\title{
Parametric Stability Margin for Multilinear Interval Control Systems
} 159594

L. H. Keel

Center of Excellence in Information Systems

Tennessee State University

Nashville, TN 37203 - 3401
S. P. Bhattacharyya

Department of Electrical Engineering

Texas A\&M University

College Station, TX 77843

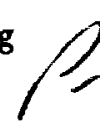

5

\section{Abstract}

Recently, a necessary and sufficient condition to determine the robust stability of a multilinear interval control system has been reported in $[1,2]$ as an extension of the well-known Box theorem [3] which deals with the linear affine case. This paper introduces a simple but computationally efficient algorithm, based on the above result, to check the robust stability of such systems. The method is also extended to find the parametric stability margin of such a system.

\section{ROBUST STABILITY}

Let $\mathbf{p}:=\left[\begin{array}{llll}p_{1} & p_{2} & \cdots & p_{l}\end{array}\right]$ a vector of real parameters lying in the interval uncertainty set

$$
\Pi:=\left\{p \mid p_{i}^{-} \leq p_{i} \leq p_{i}^{+}, \quad i=1,2, \cdots, l\right\} .
$$

Consider the polynomial

$$
\delta(s, \mathbf{p}):=\delta_{0}(\mathbf{p})+\delta_{1}(\mathbf{p}) s+\delta_{2}(\mathbf{p}) s^{2}+\cdots+\delta_{n}(\mathbf{p}) s^{n}
$$

wherein the coefficients $\delta_{i}(\mathbf{p})$ are affine multilinear functions of $p$. We shall refer to this type of polynomial as a multilinear interval polynomial. It is easy to show that any multilinear interval polynomial can be rewritten as

$$
\delta(s):=\sum_{i=1}^{m} Q_{i}(s) \prod_{j=1}^{r} P_{i j}(s)
$$

where $P_{i j}(s)$ are interval and $Q_{i}(s)$ are fixed polynomials. The necessary and sufficient condition for robust stability of the family $\delta(s)$ under the assumption that $P_{i j}(s)$ are independent is given below. Let $\mathcal{K}_{P_{i j}}(s)$ be the set of Kharitonov vertex polynomials [4] associated with the interval polynomial $P_{i j}(s)$ and $S_{P_{1 j}}\left(s, \lambda_{j}\right)$ the set of Kharitonov segment polynomials associated with interval polynomial $P_{i j}(s)$.

Theorem 1. $[1,2]$ The multilinear family $\delta(s)$ is Hurwitz stable if and only if the set of manifolds $\mathrm{M}_{i}$ for $i=1,2, \cdots, m$ are Hurwitz stable where

$$
\mathbf{M}_{l}=\sum_{i=1, i \neq l}^{m} Q_{i}(s) \prod_{j=1}^{r} \mathcal{K}_{P_{i j}}(s)+Q_{l}(s) \prod_{j=1}^{r} S_{P_{l j}}\left(s, \lambda_{j}\right)
$$

for $\lambda_{i} \in[0,1]$ for all $i$.

Now let us define the new parameter vector

$$
\underline{\lambda}:=\left[\begin{array}{llll}
\lambda_{1} & \lambda_{2} & \cdots & \lambda_{j}
\end{array}\right]
$$

then each manifold in $\mathbf{M}_{\mathbf{l}}$ can be written as the polynomial set

$$
\delta(s, \underline{\lambda}):=\delta_{0}(\underline{\lambda})+\delta_{1}(\underline{\lambda}) s+\cdots, \quad \lambda_{i} \in[0,1]
$$

where the coefficients $\delta_{i}(\underline{\lambda})$ are multilinear functions of $\lambda$. Therefore, the problem of checking robust stability of a multilinear interval control system is reduced to checking the stability of the set of multilinear polynomials shown in eq. (6), as $\underline{\lambda}$ ranges over the positive unit hypercube in the first quadrant.

Let us define the set

$$
\Delta:=\left\{\delta(s, \lambda) \mid \lambda_{i} \in[0,1]\right\} .
$$

Let $\mathrm{V}$ denote the vertices of the $\Delta$ set,

$$
\begin{aligned}
\mathrm{V}:= & \left\{\underline{\lambda} \mid \lambda_{i}=0 \text { or } \lambda_{i}=1, \text { for all } i\right\} \\
& \text { and } \Delta_{\mathrm{V}}:=\{\delta(s, \underline{\lambda}) \mid \underline{\lambda} \in \mathrm{V}\}
\end{aligned}
$$

denotes the set of vertex polynomials.

Note that $\Delta$ is a continuum of polynomials whereas $\Delta \mathrm{V}$ is a discrete set of polynomials and

$$
\Delta_{\mathbf{V}} \subset \Delta \text {. }
$$

Fixing $s=s^{*}$, we let $\Delta\left(s^{*}\right)$ denote the set of points $\delta\left(s^{*}, \underline{\lambda}\right)$ in the complex plane obtained by letting $\lambda$ range over $[0,1]$ :

$$
\Delta\left(s^{*}\right):=\left\{\delta\left(s^{*}, \underline{\lambda}\right) \mid \underline{\lambda} \in[0,1]\right\} .
$$

Likewise we have the discrete set of points in the complex plane

$$
\Delta_{\mathbf{V}}\left(s^{*}\right):=\left\{\delta\left(s^{*}, \underline{\lambda}\right) \mid \underline{\lambda} \in \mathrm{V}\right\} .
$$

We now state the well known Mapping Theorem [6]. 
Theorem 2. (Mapping Theorem [6]) Under the assumption that $\delta_{i}(\underline{\lambda})$ are affine multilinear functions of $\underline{\lambda}$

$$
\cos \Delta\left(s^{*}\right)=\cos \Delta_{\mathrm{V}}\left(s^{*}\right)
$$

for each $s^{*} \in \mathbb{C}$ where co $(\cdot)$ indicates the convex $h u l l$ of a set (.).

This theorem shows that the image set of the multilinearly parametrized interval family, evaluated at any point $s^{*}$ is contained in the convex hull of the vertices evaluated at the same point. Although the convex hull of the vertices overbounds this set we can improve the accuracy of the approximation arbitrarily by introducing additional vertices. This is illustrated in Figures 1 to 4 . The image set $\Delta\left(s^{*}\right)$ is contained in the convex hull of $\mathbf{R}\left(\mathrm{s}^{*}\right)=\operatorname{co} \Delta \mathbf{V}\left(\mathrm{s}^{*}\right)$ as shown in Figures 1 and 2. As shown in the subsequent figures (Figures 3,4 ) this approximation can be improved by decomposing $\lambda$ hypercube as a union of smaller boxes and thereby introducing additional vertices as shown in Figures A.1 and A.2.

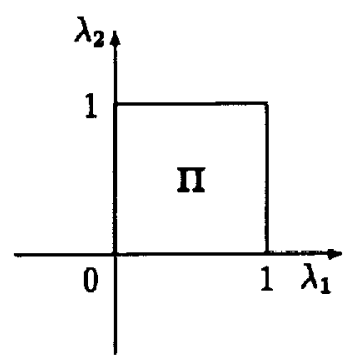

Figure A.1

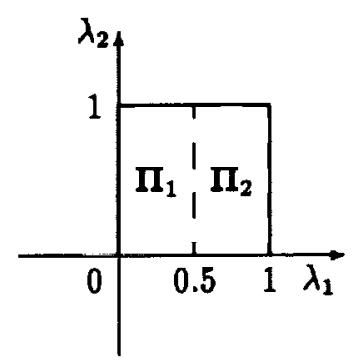

Figure A.2
From this theorem, we can easily establish the following corollary.

Corollary 1. The multilinear polynomial set $\Delta(s)$ is Hurvitz stable if the convex hull of $\Delta \mathrm{V}(s)$ is $H u r-$ witz stable. If the dependence of the coefficients $\delta_{i}(\mathbf{p})$ on $\mathrm{p}$ is linear the stability of $\Delta(s)$ is equivalent to that of the convex hull of $\Delta_{\mathrm{V}}(\mathrm{s})$.

If we define the set of convex combinations of the vertex polynomials in $\Delta_{\mathbf{V}}(s)$ by

$$
\begin{gathered}
\mathrm{E}(s):=\left\{\mu_{i j} \delta_{i}(s)+\left(1-\mu_{i j}\right) \delta_{j}(s) \mid \mu_{i j} \in[0,1]\right. \text { and } \\
\left.\delta_{i}(s), \delta_{j}(s) \in \Delta \mathrm{V}\right\},
\end{gathered}
$$

the stability of co $\Delta \mathrm{V}(s)$ is equivalent to the stability of $\mathbf{E}(\mathrm{s})$. Since the set $\mathbf{E}(\mathrm{s})$ consists of line segments joining every pair of vertices in $\Delta_{\mathrm{V}}(s)$, its stability can be easily verified by the Segment Lemma [7]. The segment lemma basically determines if the phase difference of a pair of stable vertex polynomials reaches
180 degrees at some $\omega$. If the phase difference does not reach $180^{\circ}$ for any $\omega$, the line segment joining the two vertices is Hurwitz stable. This condition is called the Phase Condition.

Using the above concepts we can easily prove the following. Let $\Delta(j \omega)$ denote the complex plane image of the set $\Delta(s)$ evaluated at $s=j \omega$.

Theorem 3. The set of multilinear polynomials $\Delta(s)$ is Hurwitz stable if i) for some $\omega \in[0, \infty)$, $0 \notin \Delta(j \omega)$ and ii) the set of all corresponding line segments $\mathrm{E}(\mathrm{s})$ is Hurwitz stable.

This theorem along with the previously given procedure for approximating the image set can be used to develop an efficient computational technique to check robust stability. We can also extend this result to the computation of the parametric stability margin of the multilinear interval control systems. This is done next.

\section{PARAMETRIC STABILITY MARGIN}

Consider a Hurwitz polynomial of the form

$$
\delta(s)=\sum_{i=1}^{m} Q_{i}(s) \prod_{j=1}^{r} P_{i j}(s)
$$

where $Q_{i}(s)$ are fixed and

$$
P_{i j}(s):=p_{i j}^{0}+p_{i j}^{1} s+p_{i j}^{2} s^{2}+\cdots
$$

Let us assume that coefficients $p_{i j}^{k}$ for $k=0,1,2, \cdots$ are subject to variations. If we assume the variations in coefficients are bounded as

$$
p_{i j}^{k} \in\left[p_{i j_{0}}^{k}-w_{i j}^{k} \epsilon, p_{i j_{0}}^{k}+w_{i j}^{k} \epsilon\right], \quad \epsilon \geq 0
$$

for a fixed value of the weights $w_{i j}$ with $p_{i j_{0}}^{k}$ being the nominal values of the parameters, then the parametric stability margin is defined as the maximum value $\epsilon^{*}$ so that the multilinear polynomial $\delta(s)$ remains Hurwitz stable for all $\epsilon \in\left[0, \epsilon^{*}\right)$.

Let us recall the manifolds shown in eq. (4). From Theorem 1, the parametric stability margin of the polynomial in eq. (15) is equivalently defined as

$$
\begin{gathered}
\min \left\{\epsilon \mid \max \left\{\epsilon \mid M_{i}^{k} \in \mathcal{H}, \forall \lambda_{i} \in[0,1]\right\},\right. \\
\left.l \in \underline{m}, k \in \underline{4}^{2 r}\right\} .
\end{gathered}
$$

Note that each manifold in $\mathbf{M}_{\mathbf{l}}$ has the form of

$$
\begin{aligned}
& Q_{1}(s) K_{P_{11}}^{i_{21}}(s) K_{P_{12}}^{i_{12}}(s) \cdots K_{P_{1 r}}^{i_{1}}(s)+\cdots \\
& +Q_{l-1}(s) K_{P_{1-11}}^{i_{1}-12}(s) K_{P_{1-12}}^{i_{1-12}}(s) \cdots K_{P_{1-1 r}}^{i_{1-1}}(s) \\
& +Q_{l}(s) S_{P_{11}}^{i_{11}}\left(s, \lambda_{1}\right) S_{P_{12}}^{i_{12}}\left(s, \lambda_{2}\right) \cdots S_{P_{1 r}}^{i_{1}}\left(s, \lambda_{r}\right) \\
& +Q_{l+1}(s) K_{P_{1+12}}^{i_{1+11}}(s) K_{P_{l+12}}^{i_{1+12}}(s) \cdots K_{P_{1+1}}^{i_{1}+1 r}(s)+\cdots
\end{aligned}
$$


where $K_{P_{i j}}^{k_{i j}}(s)$ is the $k^{\text {th }}$ Kharitonov polynomial and $S_{P_{i j}}^{k_{i j}}(s, \lambda)$ is the $k^{\text {th }}$ segment polynomial associated with an interval polynomial $P_{i j}(s)$, respectively.

Using the sufficient condition developed in Theorem 3, we can develop the following computational procedure.For some $\hat{\epsilon}$, if all the corresponding line segments in $\mathbf{E}(s)$ are Hurwitz stable for all $\epsilon \in[0, \hat{\epsilon})$, then the actual parametric stability margin $\epsilon^{*}$ is always greater than or equal to $\hat{\epsilon}$. However, if we introduce additional vertex points the approximation of the image set by the convex hull of the vertices improves and so the difference $\epsilon^{*}-\hat{\epsilon}$ becomes smaller. This permits us to compute the parametric stability margin with arbitrary accuracy depending upon how much we want to refine the approximation and how much computational burden may be taken. From the above considerations, a bisection algorithm is developed as follows:

Bisection Method: $\epsilon$

For each fixed $\epsilon$

Find all manifolds for

$\left(\lambda_{1}, \ldots, \lambda_{r}\right) \in\{(0, \cdots, 0), \cdot(1, \cdots, 1)\}$

IF all $\mathbf{E}(s)$ satisfy phase condition

THEN forward bisection

ELSE backward bisection

ENDIF

Bisection Method stops when the section piece is small enough.

\section{NUMERICAL EXAMPLES}

Example 1. Consider the feedback system shown in Figure B.

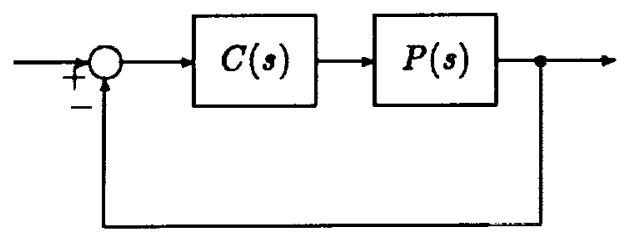

Figure B. Feedback System.

$$
\begin{aligned}
& P(s):=\frac{n(s)}{d(s)}:=\frac{n_{1} s+n_{0}}{d_{2} s^{2}+d_{1} s+d_{0}} \\
& C(S):=\frac{n_{c}(s)}{d_{c}(s)}:=\frac{s^{2}+2 s+1}{s^{4}+2 s^{3}+2 s^{2}+s}
\end{aligned}
$$

with its plant coefficient variations being bounded by

$n_{1} \in\left[n_{1}^{-}, n_{1}^{+}\right] \quad$ with the nominal value $n_{1}^{0}=.15$

$n_{0} \in\left[n_{0}^{-}, n_{0}^{+}\right] \quad$ with the nominal value $n_{0}^{0}=.95$

$d_{2} \in\left[d_{2}^{-}, d_{2}^{+}\right] \quad$ with the nominal value $d_{2}^{0}=.95$ $d_{1} \in\left[d_{1}^{-}, d_{1}^{+}\right] \quad$ with the nominal value $d_{1}^{0}=1.9$

$d_{0} \in\left[d_{0}^{-}, d_{0}^{+}\right] \quad$ with the nominal value $d_{0}^{0}=2.0$

and $\left[n_{1}^{-}, n_{1}^{+}\right]=\left[n_{1}^{0}-w_{n_{1}} \epsilon, n_{1}^{0}+w_{n_{1}} \epsilon\right]$

$\left[n_{0}^{-}, n_{0}^{+}\right]=\left[n_{0}^{0}-w_{n_{0}} \epsilon, n_{0}^{0}+w_{n_{0}} \epsilon\right]$

$\left[d_{2}^{-}, d_{2}^{+}\right]=\left[d_{2}^{0}-w_{d_{2}} \epsilon, d_{2}^{0}+w_{d_{2}} \epsilon\right]$

$\left[d_{1}^{-}, d_{1}^{+}\right]=\left[d_{1}^{0}-w_{d_{1}} \epsilon, d_{1}^{0}+w_{d_{1}} \epsilon\right]$

$\left[d_{0}^{-}, d_{0}^{+}\right]=\left[d_{0}^{0}-w_{d_{0}} \epsilon, d_{0}^{0}+w_{d_{0}} \epsilon\right]$.

Then the characteristic polynomial is given by

$$
\Pi:=\left\{d(s) d_{r}(s)+n(s) n_{c}(s)\right\}
$$

which shows that the parameters are entering into its coefficient linearly. As we stated in Corollary 1, the phase condition becomes necessary and sufficient in this case. The sets of Kharitonov polynomials associated with $n(s, \epsilon)$ and $d(s, \epsilon)$ are defined as follows:

$$
\begin{gathered}
\left\{\begin{array}{l}
K_{n}^{1}(s, \epsilon)=n_{0}^{-}+n_{1}^{-} s, \quad K_{n}^{2}(s, \epsilon)=n_{0}^{-}+n_{1}^{+} s \\
K_{n}^{3}(s, \epsilon)=n_{0}^{+}+n_{1}^{-} s, \quad K_{n}^{4}(s, \epsilon)=n_{0}^{+}+n_{1}^{+} s
\end{array}\right. \\
\left\{\begin{array}{l}
K_{d}^{1}(s, \epsilon)=d_{0}^{-}+d_{1}^{-} s+d_{2}^{+} s^{2} \\
K_{d}^{2}(s, \epsilon)=d_{0}^{-}+d_{1}^{+} s+d_{2}^{+} s^{2} \\
K_{d}^{3}(s, \epsilon)=d_{0}^{+}+d_{1}^{-} s+d_{2}^{-} s^{2} \\
K_{d}^{4}(s, \epsilon)=d_{0}^{+}+d_{1}^{+} s+d_{2}^{-} s^{2} .
\end{array}\right.
\end{gathered}
$$

Thus we need to check the phase condition of the following set of vertex pairs: $\mathbf{E}:=\mathbf{E}_{1} \cup \mathbf{E}_{2}$ where

$$
\begin{array}{cc}
\mathbf{E}_{1}:= & \left\{\left(K_{d}^{i}(s, \epsilon) d_{r}(s)+K_{n}^{j}(s, \epsilon) n_{r}(s),\right.\right. \\
& \left.\left.K_{d}^{i}(s, \epsilon) d_{r}(s)+K_{n}^{k}(s, \epsilon) n_{r}(s)\right)\right\} \\
\mathbf{E}_{2}:= & \left\{\left(K_{d}^{j}(s, \epsilon) d_{r}(s)+K_{n}^{i}(s, \epsilon) n_{r}(s),\right.\right. \\
& \left.\left.K_{d}^{k}(s, \epsilon) d_{r}(s)+K_{n}^{i}(s, \epsilon) n_{r}(s)\right)\right\} \\
\text { for } \quad & i=1,2,3,4 \text { and } \\
& (i, k) \in\{(1,2),(1,3),(2,3),(3,4)\} .
\end{array}
$$

Using a bisection method on $\epsilon$, we have the parametric stability margin:

$$
\epsilon^{*}=\max \left\{\epsilon \mid \mathbf{E} \in \mathcal{H}, \forall \epsilon \in\left[0, \epsilon^{*}\right]\right\}=0.146 .
$$

Example 2. Consider the following interconnected feedback system shown in Figure C. Let

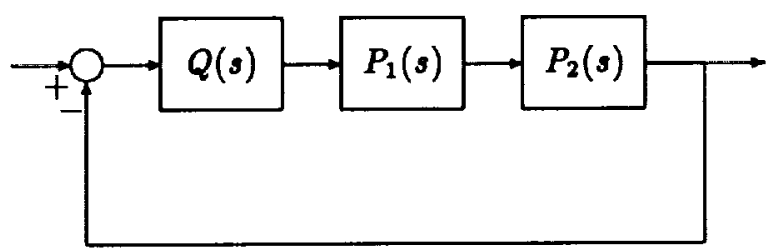

Figure C. Interconnected Feedback System.

$$
Q(s):=\frac{Q_{1}(s)}{Q_{2}(s)}=\frac{s+2}{s+1}
$$




$$
\begin{aligned}
& P_{1}(s):=\frac{P_{11}(s)}{P_{21}(s)}=\frac{s^{2}+s+1}{s^{3}+a_{2} s^{2}+4 s+a_{0}} \\
& P_{2}(s):=\frac{P_{12}(s)}{P_{22}(s)}=\frac{6.6 s^{3}+13.5 s^{2}+15.5 s+20.4}{s^{3}+b_{2} s^{2}+3.5 s+2.4}
\end{aligned}
$$

and let the set of parameters $p=\left[a_{2}, a_{0}, b_{2}\right]$ vary as follows:

$$
\begin{aligned}
a_{2} \in\left[a_{2}^{-}, a_{2}^{+}\right] & \text {with the nominal value } a_{2}^{0}=-3.0 \\
a_{0} \in\left[a_{0}^{-}, a_{0}^{+}\right] & \text {with the nominal value } a_{0}^{0}=2.0 \\
b_{2} \in\left[b_{2}^{-}, b_{2}^{+}\right] & \text {with the nominal value } b_{2}^{0}=3.5 \\
\text { where }\left[a_{2}^{-}, a_{2}^{+}\right] & =\left[a_{2}^{0}-w_{a_{2}} \epsilon, a_{2}^{0}+w_{a_{2}} \epsilon\right] \\
{\left[a_{0}^{-}, a_{0}^{+}\right] } & =\left[a_{0}^{0}-w_{a_{0}} \epsilon, a_{0}^{0}+w_{a_{0}} \epsilon\right] \\
{\left[b_{2}^{-}, b_{2}^{+}\right] } & =\left[b_{2}^{0}-w_{b_{2}} \epsilon, b_{2}^{0}+w_{b_{2}} \epsilon\right]
\end{aligned}
$$

with $\left[w_{a_{2}}, w_{a_{0}}, w_{b_{3}}\right]=[1,1,1]$. The Kharitonov vertex and segment polynomials as follows:

$$
\begin{aligned}
K_{P_{12}}^{1}(s, \epsilon) & =K_{P_{11}}^{2}(s, \epsilon)=K_{P_{11}}^{3}(s, \epsilon) \\
& =K_{P_{11}}^{4}(s, \epsilon)=P_{11}(s, \epsilon) \\
K_{P_{12}}^{1}(s, \epsilon) & =K_{P_{12}}^{2}(s, \epsilon)=K_{P_{13}}^{3}(s, \epsilon) \\
& =K_{P_{12}}^{4}(s, \epsilon)=P_{12}(s, \epsilon) \\
K_{P_{21}}^{1}(s, \epsilon) & =K_{P_{21}}^{2}(s, \epsilon) \\
& =a_{0}^{-}+4 s+a_{2}^{+} s^{2}+s^{3} \\
K_{P_{21}}^{3}(s, \epsilon) & =K_{P_{21}}^{4}(s, \epsilon) \\
& =a_{0}^{+}+4 s+a_{2}^{-} s^{2}+s^{3} \\
S_{P_{21}}^{1}(s, \epsilon) & =K_{P_{21}}^{1}(s, \epsilon) \\
S_{P_{21}}^{2}(s, \epsilon) & =S_{P_{21}}^{3}(s, \epsilon) \\
& =\lambda K_{P_{21}}^{1}(s, \epsilon)+(1-\lambda) K_{P_{21}}^{3}(s, \epsilon) \\
S_{P_{21}}^{4}(s, \epsilon) & =K_{P_{21}}^{3}(s, \epsilon) \\
K_{P_{22}}^{1}(s, \epsilon) & =K_{P_{22}}^{2}(s, \epsilon) \\
& =2.4+3.5 s+b_{2}^{+} s^{2}+s^{3} \\
K_{P_{21}}^{3}(s, \epsilon) & =K_{P_{22}}^{4}(s, \epsilon) \\
& =2.4+3.5 s+b_{2}^{-} s^{2}+s^{3} \\
S_{P_{22}}^{1}(s, \epsilon) & =K_{P_{22}}^{1}(s, \epsilon) \\
S_{P_{22}}^{2}(s, \epsilon) & =S_{P_{22}}^{3}(s, \epsilon) \\
& =\lambda K_{P_{22}}^{1}(s, \epsilon)+(1-\lambda) K_{P_{23}}^{3}(s, \epsilon) \\
S_{P_{22}}^{4}(s, \epsilon) & =K_{P_{22}}^{3}(s, \epsilon) .
\end{aligned}
$$

The sets of manifolds to be checked for stability are:

$$
\begin{aligned}
M= & \left\{Q_{1}(s) P_{11}(s, \epsilon) P_{12}(s, \epsilon)+\right. \\
& Q_{2}(s)\left[\lambda_{1} K_{P_{21}}^{1}(s, \epsilon)+\left(1-\lambda_{1}\right) K_{P_{21}}^{3}(s, \epsilon)\right] \\
& {\left.\left[\lambda_{2} K_{P_{22}}^{1}(s, \epsilon)+\left(1-\lambda_{2}\right) K_{P_{22}}^{3}(s, \epsilon)\right]\right\} }
\end{aligned}
$$

We now solve the problem of checking these manifolds by overbounding this set by the convex hull of the vertices. After eliminating all duplicated segments, we have the following line segments which need to be checked. Note that the line segments listed below are functions of $\epsilon$ and our objective is to find the maximum value of $\epsilon$ so that all $\mathbf{E}\left(s, \epsilon^{*}\right)$ remain Hurwitz for all $\epsilon \in\left[0, \epsilon^{*}\right]$.

$$
\begin{aligned}
& E_{1}(s, \epsilon)=\left(Q_{1}(s) P_{11}(s, \epsilon) P_{12}(s, \epsilon)+\right. \\
& Q_{2}(s) K_{P_{21}}^{3}(s, \epsilon) K_{P_{22}}^{3}(s, \epsilon) \text {, } \\
& Q_{1}(s) P_{11}(s, \epsilon) P_{12}(s, \epsilon)+ \\
& \left.Q_{2}(s) K_{P_{21}}^{3}(s, \epsilon) K_{P_{23}}^{1}(s, \epsilon)\right) \\
& E_{2}(s, \epsilon)=\left(Q_{1}(s) P_{11}(s, \epsilon) P_{12}(s, \epsilon)+\right. \\
& Q_{2}(s) K_{P_{21}}^{3}(s, \epsilon) K_{P_{23}}^{3}(s, \epsilon) \text {, } \\
& Q_{1}(s) P_{11}(s, \epsilon) P_{12}(s, \epsilon)+ \\
& \left.Q_{2}(s) K_{P_{21}}^{1}(s, \epsilon) K_{P_{22}}^{3}(s, \epsilon)\right) \\
& E_{3}(s, \epsilon)=\left(Q_{1}(s) P_{11}(s, \epsilon) P_{12}(s, \epsilon)+\right. \\
& Q_{2}(s) K_{P_{21}}^{3}(s, \epsilon) K_{P_{22}}^{3}(s, \epsilon), \\
& Q_{1}(s) P_{11}(s, \epsilon) P_{12}(s, \epsilon)+ \\
& \left.Q_{2}(s) K_{P_{21}}^{1}(s, \epsilon) K_{P_{23}}^{1}(s, \epsilon)\right) \\
& E_{4}(s, \epsilon)=\left(Q_{1}(s) P_{11}(s, \epsilon) P_{12}(s, \epsilon)+\right. \\
& Q_{2}(s) K_{P_{21}}^{3}(s, \epsilon) K_{P_{22}}^{1}(s, \epsilon) \text {, } \\
& Q_{1}(s) P_{11}(s, \epsilon) P_{12}(s, \epsilon)+ \\
& \left.Q_{2}(s) K_{P_{31}}^{1}(s, \epsilon) K_{P_{23}}^{3}(s, \epsilon)\right) \\
& E_{5}(s, \epsilon)=\left(Q_{1}(s) P_{11}(s, \epsilon) P_{12}(s, \epsilon)+\right. \\
& Q_{2}(s) K_{P_{21}}^{3}(s, \epsilon) K_{P_{22}}^{1}(s, \epsilon), \\
& Q_{1}(s) P_{11}(s, \epsilon) P_{12}(s, \epsilon)+ \\
& \left.Q_{2}(s) K_{P_{21}}^{1}(s, \epsilon) K_{P_{22}}^{1}(s, \epsilon)\right) \\
& E_{6}(s, \epsilon)=\left(Q_{1}(s) P_{11}(s, \epsilon) P_{12}(s, \epsilon)+\right. \\
& Q_{2}(s) K_{P_{21}}^{1}(s, \epsilon) K_{P_{23}}^{3}(s, \epsilon) \text {, } \\
& Q_{1}(s) P_{11}(s, \epsilon) P_{12}(s, \epsilon)+ \\
& \left.Q_{2}(s) K_{P_{21}}^{1}(s, \epsilon) K_{P_{23}}^{1}(s, \epsilon)\right) \text {. }
\end{aligned}
$$

Using a simple bisection method based algorithm we have

$$
\begin{aligned}
\epsilon^{*} & =\max \left\{\epsilon \mid E_{i}(s, \epsilon) \in \mathcal{H}, \forall \epsilon \in\left[0, \epsilon^{*}\right], i=1, \cdots, 6\right\} \\
& =0.6305 .
\end{aligned}
$$

\section{CONCLUDING REMARKS}

A simple technique to determine the parametric stability margin for multilinearly dependent interval control system has been given. The method is based on the multilinear generalization of the Box theorem. The method is powerful and reduces computational burden significantly. In fact, if used in conjunction with the Segment Lemma it completely eliminates frequency sweeping. Furthermore, by adding additional vertices one may achieve an arbitrarily high level of accuracy.

\section{References}

[1] H. Chapellat, L. H. Keel, and S. P. Bhattacharyya, "Stability margins for multilinear in- 
terval control systems," in Proceedings of the 30th IEEE Conference on Decision and Control, (Brighton, U.K.), December 1991.

[2] S. P. Bhattacharyya and L. H. Keel, "Robust stability and control of linear and multilinear interval systems," Control and Dynamic Systems, vol. 51, pp. 31 - 78, 1992. Academic Press.

[3] H. Chapellat and S. P. Bhattacharyya, "A generalization of Kharitonov's theorem: robust stability of interval plants," IEEE Transactions on Automatic Control, vol. AC - 34, pp. 306 - 311, March 1989.

[4] V. L. Kharitonov, "Asymptotic stability of an equilibrium position of a family of systems of linear differential equations," Differential Uravnen, vol. 14, pp. 2086 - 2088, 1978.

[5] A. Katbab and E. I. Jury, "Robust Schur-stability of control systems with interval plants," International Journal of Control, vol. 51, no. 6, pp. 1343 $-1352,1990$.

[6] L. A. Zadeh and C. A. Desoer, Linear Systems Theory. New York: McGraw Hill, 1963.

[7] H. Chapellat and S. P. Bhattacharyya, "An alternative proof of Kharitonov's theorem," IEEE Transactions on Automatic Control, vol. AC - 34, pp. 448 - 450, April 1989.

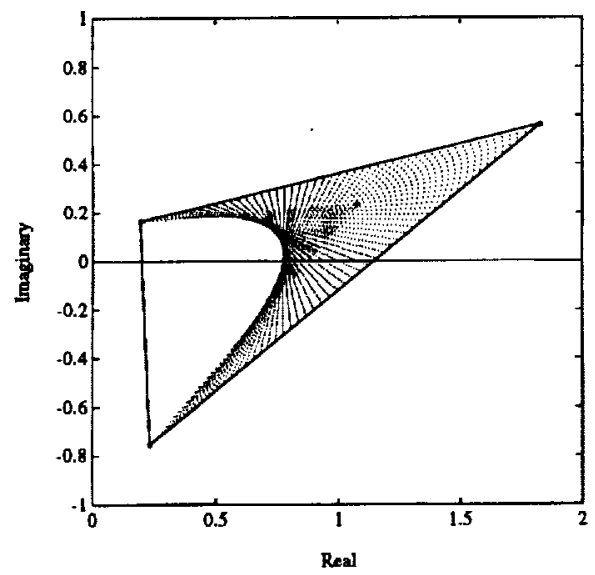

Figure 1: Image Set $(\omega=0.85)$

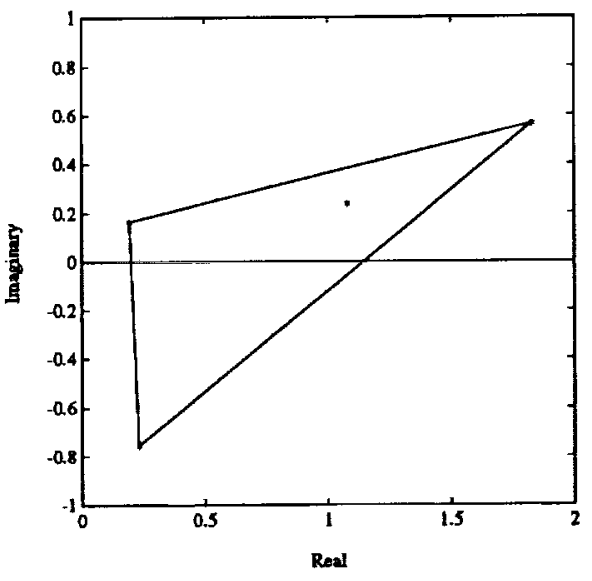

Figure 2: Convex Hull $(\omega=0.85)$

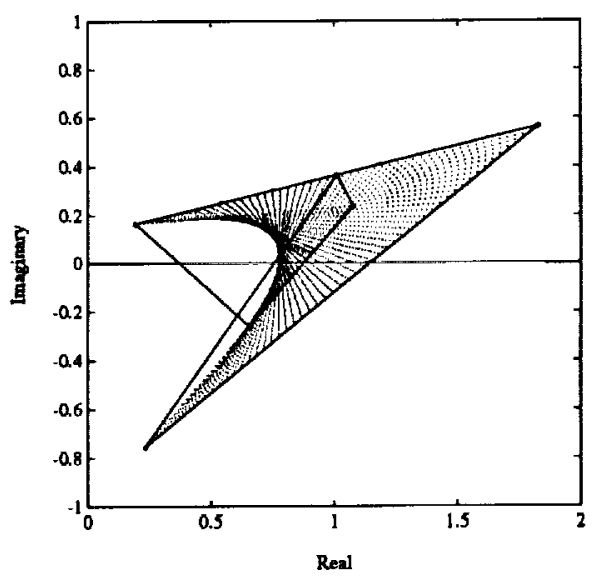

Figure 3: Image Set $(\omega=0.85)$

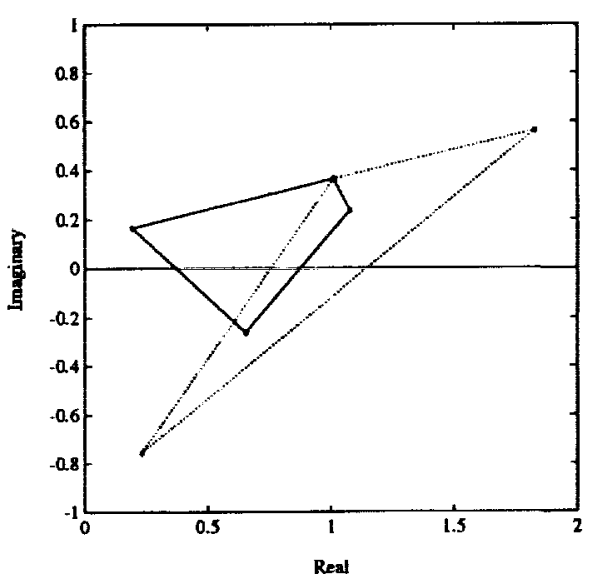

Figure 4: Convex Hull $(\omega=0.85)$ 